\title{
Achieving the gold standard in dental aesthetics: manufacturing individual prostheses by measuring mandibular kinematics
}

Lately, more and more dental equipment companies have introduced devices to measure the movement of the lower jaw to increase the customization of prostheses. Many years ago I came to familiarize myself with such a mandibular kinematics recording device, JMAnalyser+ from Zebris Medical GmbH. I used that device to make measurements in a batch of patients under a multicenter research grant whose director was my father.

During the IDS 2017, I noticed a multitude of devices to measure mandibular kinematics. I took my father to the Zebris Medical GmbH stand (Am Galgenbuehl 14, D-88316 Isny, Germany) we learned from engineer Wolfgang Brunner, one of the owners, that his company manufactures a wide range of devices for the recording of mandibular kinematics at SAMDental, KaVo, Dentsply / Sirona, Schütz Dental $\mathrm{GmbH}$, etc.

Out of the products on display we noticed the new JMAnalyser+ - Face Imager multifunctional device on an adjustable mobile holder.

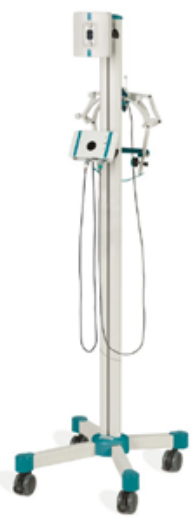

The JMAnalyser+ - Face Imager multifunctional system (Zebris Medical GmbH, D-88316 Isny, Germany) https://www.zebris.de/en/dental/products-solutions/

The JMAnalyser+ measuring system is manufactured according to the method of measuring the travel time of ultrasound impulses. It is made up of a face bow with integrated receiver modules and a balanced mandibular sensor. The sensor is fastened on the paraocclusal lower jaw attachment by means of a magnetic holder. The measurement data are transmitted to the evaluation computer cable-free via a Bluetooth or USB interface. The basic unit can be comfortably worn by the patient via a retaining strap to the neck.

The system records all degrees of the lower jaw mobility contact-free. The measuring range allows functional and occlusal analysis, recording of parameters to adjust the mechanical and virtual articulations required for individual prosthesis and occlusal splints, determination of the neuromuscular mandibular relation, and electronic position analysis of condyles (EPA).

The system is ready for export of individual motion data and forms an interface in the digital workflow. The patented bite forks connect actual motion data to dental surfaces of intraoral scans. The movements are then exported through XML files to a CAD / CAM or CBCT systems.

The evaluation software WINJAW+ convinces via a structured and modular surface that works with the wireless pedal or PC keyboard. This software is based on the integrated database and the individual measuring modules configured individually according to the user's requirements. Each movement is recorded in real-time on the screen and can be repeated at any time.

The Face Imager module identifies the facial aesthetics of the patient using the camera and intuitive software to facilitate manufacturing functional prosthesis and occlusal splints.

The additionally available integrated 4 channel EMG module allows the analysis of the muscle action potentials using bipolar surface electrodes. The biopotentials collection takes place on both sides with integrated electrode cables with integrated amplifiers. The system allows functional tests of the anterior temporalis and masseter muscle groups. The EMG activity is accurately and reliably detected without cable artifacts.

To conclude I can assure you that the JMAnalyser+ - Face Imager multifunctional system convinces by its simple operability and compact size, as it is ready to help you record the exact mandibular kinematics required to manufacture individual functional prostheses that fully satisfy your patients' aesthetic requirements.

Florin - Eugen Constantinescu DMD, PhD Student

Editorial Director, Product News

DOI: 10.25241/stomaeduj.2017.4(3).prodnews.1 